\title{
Phenome-wide association study identifies marked increased in burden of comorbidities in African Americans with systemic lupus erythematosus
}

\author{
April Barnado ${ }^{1 *}$, Robert J. Carroll², Carolyn Casey ${ }^{3}$, Lee Wheless ${ }^{4}$, Joshua C. Denny ${ }^{1,2}$ and Leslie J. Crofford
}

\begin{abstract}
Background: African Americans with systemic lupus erythematosus (SLE) have increased renal disease compared to Caucasians, but differences in other comorbidities have not been well-described. We used an electronic health record (EHR) technique to test for differences in comorbidities in African Americans compared to Caucasians with SLE.

Methods: We used a de-identified EHR with 2.8 million subjects to identify SLE cases using a validated algorithm. We performed phenome-wide association studies (PheWAS) comparing African American to Caucasian SLE cases and African American SLE cases to matched non-SLE controls. Controls were age, sex, and race matched to SLE cases. For multiple testing, a false discovery rate (FDR) $p$ value of 0.05 was used.

Results: We identified 270 African Americans and 715 Caucasians with SLE and 1425 matched African American controls. Compared to Caucasians with SLE adjusting for age and sex, African Americans with SLE had more comorbidities in every organ system. The most striking included hypertension odds ratio $(\mathrm{OR})=4.25, \mathrm{FDR} p=5.49 \times 10^{-15}$; renal dialysis $\mathrm{OR}=10.90, \mathrm{FDR} p=8.75 \times 10^{-14}$; and pneumonia $\mathrm{OR}=3.57, \mathrm{FDR} p=2.32 \times 10^{-8}$. Compared to the African American matched controls without SLE, African Americans with SLE were more likely to have comorbidities in every organ system. The most significant codes were renal and cardiac, and included renal failure $\left(\mathrm{OR}=9.55, \mathrm{FDR} p=2.26 \times 10^{-40}\right)$ and hypertensive heart and renal disease $\left(\mathrm{OR}=8.08, \mathrm{FDR} p=1.78 \times 10^{-22}\right)$. Adjusting for race, age, and sex in a model including both African American and Caucasian SLE cases and controls, SLE was independently associated with renal, cardiovascular, and infectious diseases (all $p<0.01$ ).

Conclusions: African Americans with SLE have an increased comorbidity burden compared to Caucasians with SLE and matched controls. This increase in comorbidities in African Americans with SLE highlights the need to monitor for cardiovascular and infectious complications.
\end{abstract}

Keywords: Systemic lupus erythematosus, Electronic health records, Phenome-wide association study, Health disparities, Racial disparities

\footnotetext{
*Correspondence: april.barnado@vanderbilt.edu

${ }^{1}$ Department of Medicine, Vanderbilt University Medical Center, 1161 21st

Avenue South, T3113 MCN, Nashville, TN 37232, USA

Full list of author information is available at the end of the article
} 


\section{Background}

Health disparities are defined as "differences in the incidence, prevalence, mortality and burden of diseases and other adverse health conditions that exist among specific population groups in the US" [1]. Among rheumatic diseases, systemic lupus erythematosus (SLE) has one of the highest mortality rates and highest rates of health disparity [2]. SLE disproportionately affects African Americans, particularly female African Americans, who have nearly threefold higher incidence of SLE compared to Caucasians [3]. Female African Americans also have a younger age of onset and increased rates of renal disease compared to Caucasians [3, 4]. Female African Americans in the USA have the highest SLE mortality rates [5, 6]. Studies, however, have not fully examined differences in comorbidities in African Americans compared to Caucasians with SLE.

Racial disparities in SLE have mainly been studied using cohort and administrative database studies. Cohort studies typically have focused on SLE-related disease measures such as disease activity and may not capture other important comorbidities. Alternatively, administrative studies may not capture detailed data on a patient's SLE disease course or comorbidities. Therefore, studies have not fully examined the impact of both the SLE disease course and comorbidities on outcomes. Electronic health records (EHRs) serve as an efficient and costeffective discovery tool [7-9] to provide detailed data on both a patient's SLE disease course and comorbidities. One method to harness the power of the longitudinal, clinical data in the EHR is the phenome-wide association study (PheWAS). Similar to the way a genome-wide association study (GWAS) scans across the genome, a PheWAS scans across diseases in the EHR, using aggregations of billing codes. PheWAS have uncovered novel genetic associations in multiple autoimmune diseases [10-13] and have found novel phenotypes with autoantibodies in rheumatoid arthritis [14-16]. PheWAS has also been validated across multiple EHRs and using orthogonal methods $[11-13,17,18]$. To the best of our knowledge, PheWAS have not been used in SLE to examine differences in comorbidities between African Americans and Caucasians with SLE. We hypothesized that PheWAS could take advantage of the longitudinal data in the EHR to systematically test for differences in comorbidities that would inform racial disparities in SLE.

\section{Methods}

\section{Study population}

After approval from the Institutional Review Board of Vanderbilt University Medical Center (VUMC), we identified potential SLE subjects in Vanderbilt's Synthetic Derivative [19]. VUMC is a regional, tertiary care medical center. The Synthetic Derivative is a de-identified version of the EHR with over 2.8 million subjects with longitudinal data over several decades. The Synthetic Derivative contains all available information in the EHR such as diagnostic and procedure codes, demographics, inpatient and outpatient notes (including both subspecialty and primary care), laboratory values, radiology and pathology results, and medication orders. Outside records are not available in the Synthetic Derivative. The Synthetic Derivative is composed of approximately equal numbers of male and female individuals who are predominantly Caucasian (81\%), reflecting the patient population of VUMC.

To identify SLE patients within the Synthetic Derivative, we used our validated EHR algorithm [20] of $\geq 4$ counts of the SLE ICD-9 code (710.0) and a positive anti-nuclear antibody (ANA) with a titer $\geq 1: 160$ while excluding ICD-9 codes for systemic sclerosis (710.1) and dermatomyositis (710.3). This previously described algorithm [20] has a positive predictive value (PPV) of $89 \%$ and sensitivity of $86 \%$.

Non-SLE controls were defined as subjects within the Synthetic Derivative who did not have ICD-9 codes for the 710." heading "Diffuse diseases of connective tissue," 714." heading of "Rheumatoid Arthritis and other inflammatory polyarthropathies," or ICD-10 codes under M05." ("Rheumatoid Arthritis with rheumatoid factor"), M06." ("Other rheumatoid arthritis"), M32." ("SLE"), M33." ("Dermatopolymyositis"), M34." ("Systemic sclerosis"), M35." "'Other systemic involvement of connective tissue"), and M36." ("Systemic disorders of connective tissue in diseases classified elsewhere"). Controls were age ( \pm 5 years), race, and sex matched in a 5:1 ratio to SLE cases to maximize power while allowing for close matching. Controls were "medical home" patients [21] who received longitudinal care at VUMC, defined as three outpatient visits within 5 years, to ensure similar density of records to the SLE cases. We examined age at time of analysis, age at first use of the SLE ICD-9 code, sex, and race. Race was derived from the EHR, which is a mixture of self-report and administrative entry. Prior studies have validated that these EHR race assignments reflect self-report and genetic ancestry [22]. Due to the small numbers of subjects with SLE who were Asian $(n=25)$ or Hispanic $(n=30)$, analyses were restricted to Caucasian and African American subjects with SLE, as PheWAS requires models to have at least 20 subjects for a particular code to be used in the model.

\section{Phenome-wide association studies and statistics}

In PheWAS, the 18,000 ICD-9 codes are collapsed into 1800 PheWAS codes that represent distinct clinical diagnoses. The ICD-9 codes that are mapped to PheWAS codes are version 1.2 and available at http://phewascata log.org. To be a case, we required the subject to have at least two instances of the PheWAS code on different 
days, at least 1 day apart. A subject is a control if there are no instances of the ICD-9 code for the given disease or related diseases. Subjects having only one instance of the code are excluded to eliminate the possibility of coding errors or preliminary diagnoses that may be ultimately ruled out [23]. For each PheWAS code, an unconditional logistic regression model was created with the option to add covariates with odds ratios (ORs) and 95\% confidence intervals (CIs) reported. There must be at least 20 cases $[10,11]$ for the code to be used in the model. Analyses were performed and graphed in the PheWAS package [23] in $\mathrm{R}$ version 3.2.5. We performed (1) PheWAS comparing African Americans to Caucasians with SLE, adjusting for age and sex, (2) PheWAS comparing African American SLE cases to non-SLE, matched controls, and (3) PheWAS comparing Caucasian SLE cases to non-SLE, matched controls. We adjusted for multiple hypotheses testing using a false discovery rate (FDR) of 0.05 . There were 478 testable phenotypes for the African American vs. Caucasian SLE PheWAS, 430 for the African American SLE cases vs. matched controls PheWAS, and 732 for the Caucasian SLE cases vs. matched controls. For the most significant codes, we performed conditional logistic regression using SLE cases and matched controls (including both African Americans and Caucasians) to calculate an OR and 95\% CI for the association between the codes and SLE, adjusting for age, sex, and race. We assessed for differences in demographics in African Americans vs. Caucasians with SLE using the Mann-Whitney $U$ test for continuous variables, as there were non-normal distributions in the data, and the chi-square or Fisher's exact test for categorical variables. Two-sided $p$ values $<0.05$ were considered to indicate statistical significance. Analyses were conducted using IBM SPSS software, version 24.0 (SPSS).

\section{Results}

\section{Study population}

Using our validated algorithm [20], we identified 1097 subjects with SLE including 3 with missing race/ethnicity data, 2 as "other" and 52 as "unknown" race/ethnicity, 25 Asians, 30 Hispanics, 270 African Americans, and 715 Caucasians. We then restricted analyses to the African American and Caucasian subjects with SLE. Both African American and Caucasian subjects with SLE were predominantly female ( $89 \%$ vs. $90 \%, p=0.83$ ). Compared to Caucasians, African Americans were younger (44 \pm 17 vs. $53 \pm 17, p<0.001)$ and had an earlier age at first SLE code (35 \pm 16 vs. $43 \pm 17, p<0.001$ ) with similar mean years of EHR follow up ( $9 \pm 5$ vs. $10 \pm 5, p=0.10)$ (Table 1$)$.

\section{PheWAS of Caucasians and African Americans with SLE} In the PheWAS comparing Caucasians to African Americans with SLE, adjusting for current age and sex,
Table 1 Demographics of African Americans and Caucasians with SLE

\begin{tabular}{llll}
\hline Demographics & $\begin{array}{l}\text { African Americans } \\
(n=270)\end{array}$ & $\begin{array}{l}\text { Caucasians } \\
(n=715)\end{array}$ & $p$ value $^{a}$ \\
\hline $\begin{array}{l}\text { Current age, } \\
\text { mean } \pm \text { SD }\end{array}$ & $44 \pm 17$ & $53 \pm 17$ & $<0.001$ \\
$\begin{array}{l}\text { Age at first SLE code } \\
(710.0), \text { mean } \pm \text { SD }\end{array}$ & $35 \pm 16$ & $43 \pm 17$ & $<0.001$ \\
$\begin{array}{l}\text { Female (\%) } \\
\begin{array}{l}\text { Years of follow up in } \\
\text { the EHR, mean } \pm \text { SD }\end{array}\end{array}$ & $99 \pm 5$ & 90 & 0.83 \\
\hline
\end{tabular}

SLE systemic lupus erythematosus, EHR electronic health record

${ }^{a}$ Mann-Whitney U or Fisher's exact test

there were 163 codes that met the FDR of 5\% (Additional file 1: Table S1). Compared to Caucasians, African Americans had codes for more comorbidities in every organ system (Fig. 1). The most significant codes, seen more frequently in the African Americans with SLE, were cardiac and renal. The most significant cardiac code was hypertension (OR $=4.25,95 \%$ CI 3.055.92, FDR $p=5.49 \times 10^{-15}$ ). Other significant cardiac codes covered a range of cardiovascular comorbidities including congestive heart failure (CHF) (FDR $p=5.63$ $\left.\times 10^{-6}\right)$, cerebrovascular disease (CVD) (FDR $p=4.23 \times$ $10^{-4}$ ), thromboembolism (FDR $p=4.47 \times 10^{-4}$ ), peripheral vascular disease (PVD) (FDR $p=0.04)$, coronary artery disease (CAD) (FDR $p=0.04$ ), and arrhythmias (FDR $p=0.04)$ (Table 2). The most significant renal code was renal dialysis $(\mathrm{OR}=10.90,6.11-19.48$, FDR $p=8.75 \times 10^{-14}$ ) (Table 2). Other codes included acute renal failure (FDR $p=1.05 \times 10^{-11}$ ), chronic renal failure $\left(\mathrm{FDR} p=7.08 \times 10^{-10}\right.$ ), renal transplant (FDR $p=2.32 \times 10^{-8}$ ), and nephritis related codes. In addition to cardiovascular and renal disease, African Americans with SLE were also more likely to have codes related to infection. The most significant code was pneumonia $(\mathrm{OR}=3.57,2.57$ 5.38, FDR $p=2.32 \times 10^{-8}$ ) with other significant codes related to cellulitis, pyelonephritis, and bacteremia (Table 2).

Compared to Caucasians with SLE, African Americans with SLE were more likely to have codes related to SLE criteria (Additional file 1: Figure S1 and Table S2). The most significant codes were pleurisy/pleural effusion $\left(\mathrm{OR}=4.39,2.92-6.62\right.$, FDR $\left.p=5.53 \times 10^{-11}\right)$ and nephritis; nephrosis; renal sclerosis $(\mathrm{OR}=3.03,2.10-4.37$, FDR $p=5.60 \times 10^{-8}$ ). African Americans had more codes related to serositis including pericarditis (FDR $p=$ $5.52 \times 10^{-4}$ ) and hematologic abnormalities such as thrombocytopenia (FDR $p=7.03 \times 10^{-}{ }^{4}$ ). African Americans were also more likely to have codes related to arthritis including joint effusions (FDR $p=8.41 \times 10^{-4}$ ) and neuropsychiatric involvement with encephalopathy $(\mathrm{FDR} p=0.04)$. 


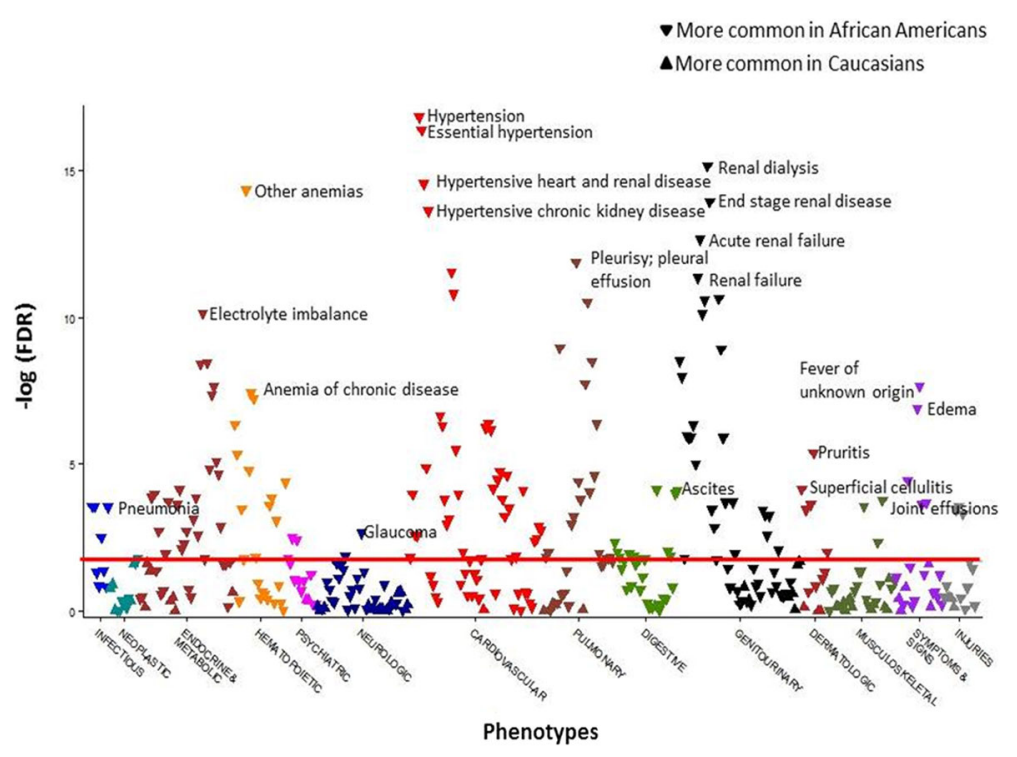

Fig. 1 Increased comorbidities across all organ systems in African Americans with systemic lupus erythematosus (SLE) compared to Caucasians using phenome-wide association studies (PheWAS). The $x$ axis represents the PheWAS codes that are mapped to ICD-9 codes, organized and color-coded by organ system. The y axis represents the level of significance. Each triangle represents a PheWAS code. African Americans are the reference group. Triangles pointing down represent codes more common in African Americans. Triangles pointing up represent codes more common in Caucasians. The PheWAS was adjusted for age and sex, and the horizontal red line represents the false discovery rate (FDR) of 0.05 . There were 163 codes that met the FDR of 0.05 . African Americans with SLE had more codes compared to Caucasians with SLE for comorbidities across all organ systems. The most significant codes for each organ system are labeled

\section{PheWAS of African Americans with SLE and African American controls}

To examine the increased cardiac, renal, and infectious comorbidities seen in the African Americans with SLE, we compared African Americans with SLE to African Americans without SLE as our controls, given known health disparities in African Americans. We identified 1425 control subjects who were age ( \pm 5 years), sex, and race matched in a 5:1 ratio to the 270 African American SLE case subjects. African American SLE case subjects and their matched controls had similar mean current age ( $44 \pm 17$ vs. $44 \pm 16, p=0.97)$ and were predominantly female (89\% vs. 93\%, $p=0.11)$.

Using PheWAS to compare African Americans with SLE to matched controls, there were 213 codes that met the FDR of 5\%. Compared to controls, African American SLE case subjects had more codes related to comorbidities in every organ system (Fig. 2a). African American SLE case subjects were more likely to have codes related to cardiovascular, renal, and infectious diseases. The most significant renal code was renal failure $(\mathrm{OR}=9.55$, 6.91-13.18, FDR $p=2.26 \times 10^{-40}$ ) with other significant codes including renal dialysis, end-stage renal disease, and renal transplant (Additional file 1: Table S3). The most significant cardiac code included hypertensive heart and/or renal disease $(\mathrm{OR}=8.08,5.39-12.11$, FDR $\left.p=1.78 \times 10^{-22}\right)$. Other significant codes covered a range of cardiovascular disease comorbidities including thromboembolism (FDR $p=4.74 \times 10^{-13}$ ), $\mathrm{CHF}$ $\left(\right.$ FDR $\left.p=4.17 \times 10^{-10}\right)$, PVD $\left(\right.$ FDR $\left.p=8.84 \times 10^{-8}\right)$, atrial fibrillation $(\mathrm{AF})\left(\mathrm{FDR} p=1.91 \times 10^{-4}\right)$, and CAD (FDR $p=0.02$ ). The most significant infection code was pneumonia OR $=5.77$ (3.97-8.39, FDR $p=8.66$ $\left.\times 10^{-19}\right)$. Other significant codes included sepsis $\left(\right.$ FDR $\left.p=7.49 \times 10^{-9}\right)$, candidiasis $\left(\right.$ FDR $\left.p=1.34 \times 10^{-7}\right)$, and cellulitis $\left(\right.$ FDR $\left.p=5.41 \times 10^{-4}\right)$.

As SLE cases had significantly longer EHR follow up compared to controls, we conducted a sensitivity analysis adjusting for years of EHR follow up to see if the longer follow up could account for the higher risk of comorbidities in the SLE cases. Adjusting for years of follow up, case subjects with SLE were still more likely to have codes related to cardiovascular, renal, and infectious diseases. The most significant renal, cardiovascular, and infectious diseases were relatively unchanged with renal failure $(\mathrm{OR}=10.12,95 \%$ CI 7.23-14.17, FDR $p=$ $\left.7.33 \times 10^{-39}\right)$, hypertensive heart and/or renal disease $\left(\mathrm{OR}=8.51,5.59-12.95\right.$, FDR $\left.p=6.90 \times 10^{-22}\right)$, and pneumonia $\left(\mathrm{OR}=6.09,4.13-9.00\right.$, FDR $\left.p=2.11 \times 10^{-19}\right)$.

Compared to matched controls, African American subjects with SLE had more codes related to American College of Rheumatology (ACR) SLE criteria [24] with the most significant codes related to renal criteria including nephritis; nephrosis; renal sclerosis $(\mathrm{OR}=50.53$, 
Table 2 Selected cardiac, renal, and infectious codes from the PheWAS of African Americans compared to Caucasians with SLE

\begin{tabular}{|c|c|c|c|c|}
\hline $\begin{array}{l}\text { PheWAS code name } \\
\text { (code number) }\end{array}$ & $\begin{array}{l}\text { Phenotype present }(\geq 2 \\
\text { instances of PheWAS code) }\end{array}$ & $\begin{array}{l}\text { Phenotype absent ( } 0 \text { instances } \\
\text { of PheWAS code) }\end{array}$ & $\begin{array}{l}\text { Adjusted odds ratio for current } \\
\text { age and sex }(95 \% \mathrm{Cl})\end{array}$ & $\begin{array}{l}\text { False discovery } \\
\text { rate } p^{b}\end{array}$ \\
\hline \multicolumn{5}{|l|}{ Selected cardiac codes } \\
\hline Hypertension (401) & 423 & 494 & $\begin{array}{l}\text { African American, } 4.25 \\
\text { (3.05-5.92) } \\
\text { Caucasian, } 1.00 \text { (ref) }\end{array}$ & $5.49 \times 10^{-15}$ \\
\hline $\begin{array}{l}\text { Other forms of chronic heart } \\
\text { disease (414) }\end{array}$ & 30 & 790 & $9.10(3.94-20.98)$ & $2.69 \times 10^{-6}$ \\
\hline Congestive heart failure (428) & 86 & 702 & $3.53(2.15-5.78)$ & $5.63 \times 10^{-6}$ \\
\hline Cerebrovascular disease (433) & 85 & 841 & $2.80(1.69-4.65)$ & $4.23 \times 10^{-4}$ \\
\hline $\begin{array}{l}\text { Other venous embolism and } \\
\text { thrombosis ( } 452 \text { ) }\end{array}$ & 89 & 741 & $2.58(1.62-4.13)$ & $4.47 \times 10^{-4}$ \\
\hline Heart valve disorders (395) & 68 & 794 & $2.89(1.69-4.95)$ & $5.52 \times 10^{-4}$ \\
\hline $\begin{array}{l}\text { Pulmonary embolism and } \\
\text { infarction, acute (415.11) }\end{array}$ & 28 & 785 & $3.73(1.69-8.23)$ & $4.03 \times 10^{-3}$ \\
\hline Cardiomyopathy (425) & 27 & 823 & $2.87(1.29-6.41)$ & 0.03 \\
\hline $\begin{array}{l}\text { Peripheral vascular disease } \\
\text { (443.9) }\end{array}$ & 25 & 738 & $3.16(1.27-7.90)$ & 0.04 \\
\hline Atherosclerosis (440) & 24 & 738 & $2.99(1.22-7.37)$ & 0.04 \\
\hline Cardiac dysrhythmias (427) & 171 & 684 & $1.60(1.08-2.36)$ & 0.04 \\
\hline \multicolumn{5}{|l|}{ Selected renal codes } \\
\hline Renal dialysis (585.31) & 73 & 600 & $10.90(6.11-19.48)$ & $8.75 \times 10^{-14}$ \\
\hline End stage renal disease (585.32) & 76 & 600 & $8.57(4.97-14.79)$ & $7.15 \times 10^{-13}$ \\
\hline $\begin{array}{l}\text { Hypertensive chronic kidney } \\
\text { disease (401.22) }\end{array}$ & 103 & 494 & $6.63(4.08-10.77)$ & $1.24 \times 10^{-12}$ \\
\hline Acute renal failure (585.1) & 149 & 600 & $4.43(2.98-6.60)$ & $1.05 \times 10^{-11}$ \\
\hline Chronic renal failure (CKD) (512) & 169 & 600 & $3.64(2.49-5.33)$ & $7.08 \times 10^{-10}$ \\
\hline Kidney replaced by transplant (587) & 49 & 600 & $7.67(3.98-14.78)$ & $2.32 \times 10^{-8}$ \\
\hline $\begin{array}{l}\text { Nephritis; nephrosis; renal sclerosis } \\
\text { (512.7) }\end{array}$ & 188 & 600 & $3.03(2.10-4.37)$ & $5.60 \times 10^{-8}$ \\
\hline Glomerulonephritis (580.1) & 90 & 600 & $4.10(2.53-6.65)$ & $1.66 \times 10^{-7}$ \\
\hline \multicolumn{5}{|l|}{ Selected infection codes } \\
\hline Pneumonia (480) & 128 & 701 & $3.57(2.37-5.38)$ & $2.32 \times 10^{-8}$ \\
\hline $\begin{array}{l}\text { Superficial cellulitis and abscess } \\
\text { (681) }\end{array}$ & 92 & 793 & $2.56(1.61-4.06)$ & $4.30 \times 10^{-4}$ \\
\hline Pyelonephritis (590) & 29 & 538 & $4.45(2.03-9.74)$ & $9.20 \times 10^{-4}$ \\
\hline Bacteremia (038.3) & 58 & 894 & $2.83(1.62-4.95)$ & $1.18 \times 10^{-3}$ \\
\hline Candidiasis (112) & 72 & 806 & $2.57(1.54-4.29)$ & $1.23 \times 10^{-3}$ \\
\hline $\begin{array}{l}\text { Cellulitis and abscess of trunk } \\
(681.7)\end{array}$ & 22 & 793 & $5.62(2.18-14.45)$ & $1.41 \times 10^{-3}$ \\
\hline Sepsis and SIRS (994) & 66 & 888 & $1.88(1.10-3.20)$ & 0.05 \\
\hline
\end{tabular}

SLE systemic lupus erythematosus, PheWas phenome-wide association studies, SIRS systemic inflammatory response syndrome

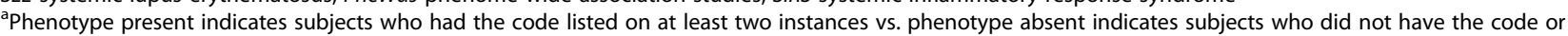
related codes. Subjects with one instance of a code are excluded, so the total number of subjects for each PheWAS code does not add up to the 1097 subjects with SLE

${ }^{\mathrm{b}}$ Codes listed met the false discovery rate of 0.05

29.40-86.86, FDR $p=4.77 \times 10^{-43}$ ) and glomerulonephritis $\left(\mathrm{OR}=72.36,32.04-163.39, \mathrm{FDR} p=2.88 \times 10^{-23}\right)$. Other significant codes represented serositis, arthritis, and hematologic and neuropsychiatric involvement (Additional file 1: Table S4).

\section{PheWAS of Caucasians with SLE and Caucasian controls}

We compared 715 Caucasian subjects with SLE to 3731 controls who were age ( \pm 5 years), sex, and race matched in a 5:1 ratio. Caucasian subjects with SLE and their matched controls had similar mean current 
- More common in African American controls

- More common in African American SLE cases

\section{a}

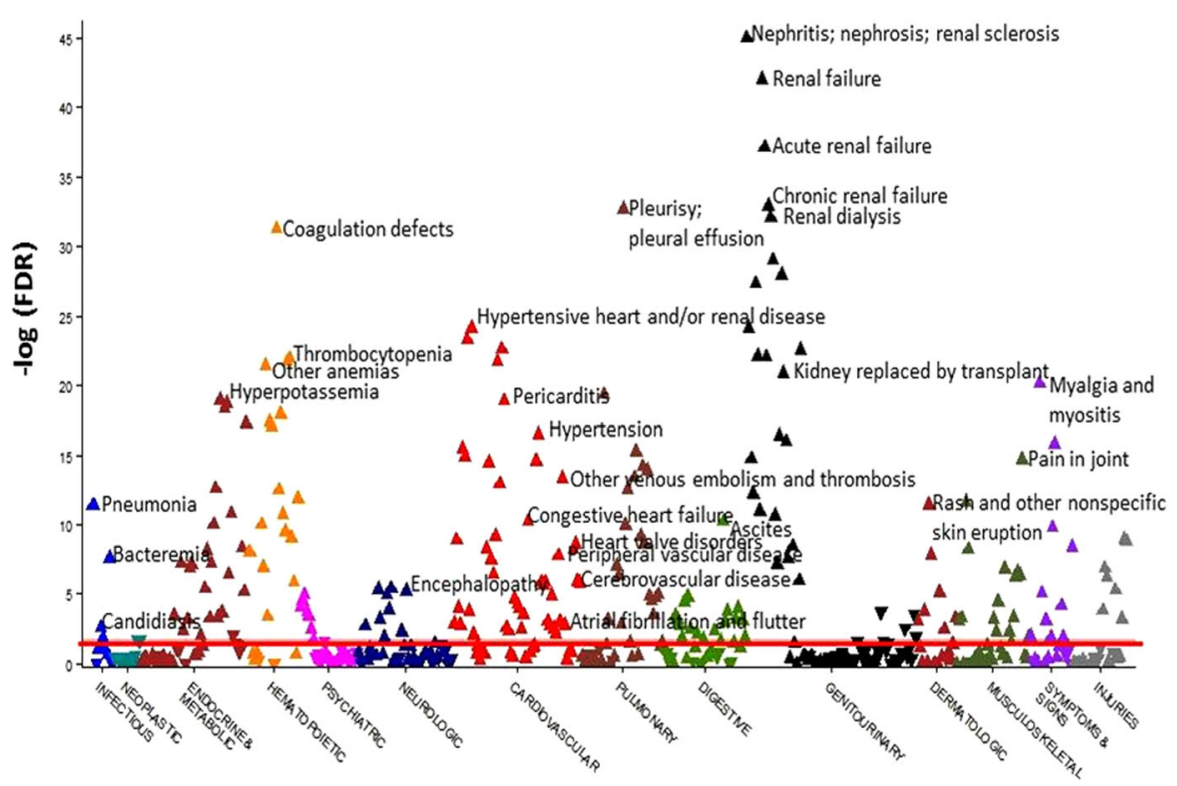

Phenotypes

b

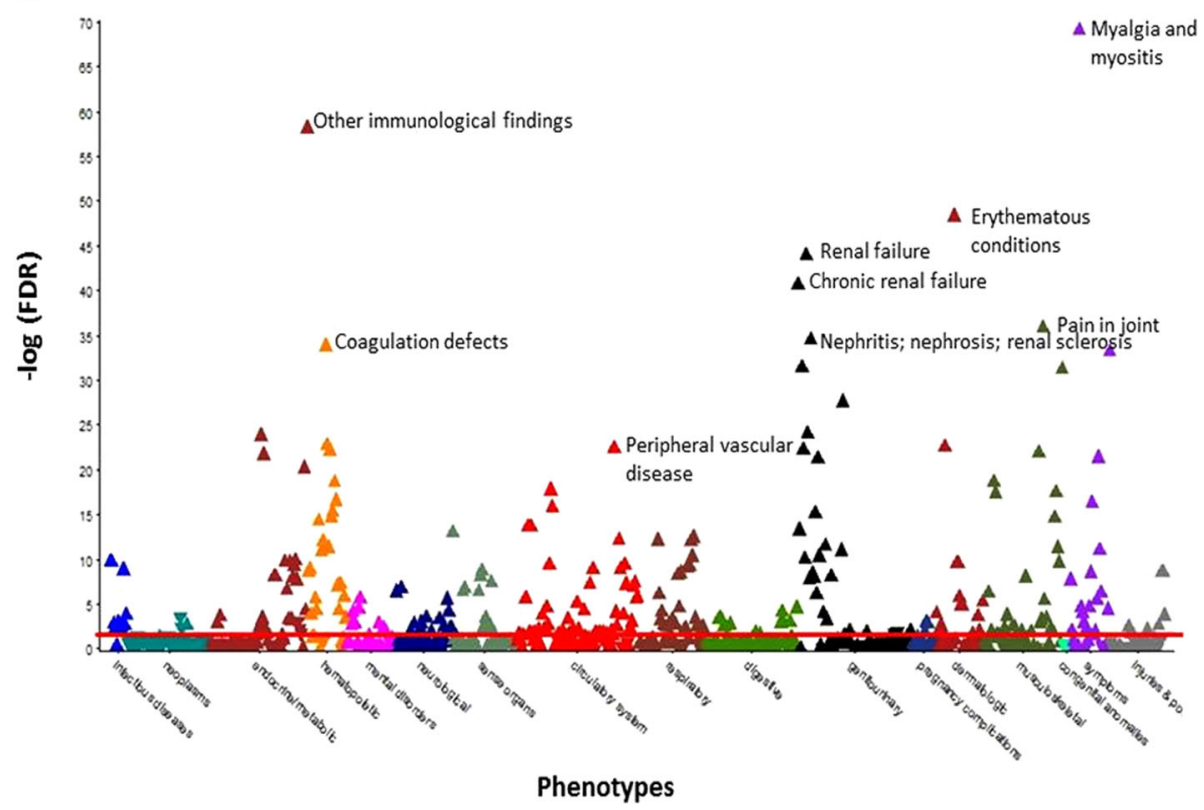

Fig. 2 Increased comorbidities in case subjects with systemic lupus erythematosus (SLE) compared to matched controls using phenome-wide association studies (PheWAS). The $x$ axis represents the PheWAS codes that are mapped to ICD-9 codes, organized and color-coded by organ system. The $y$ axis represents the level of significance. Each triangle represents a PheWAS code. Controls are the reference group and were age ( \pm 5 years), sex, and race matched. Triangles pointing up represent codes more common in subjects with SLE. Triangles pointing down represent codes more common in non-SLE matched controls. The horizontal red line represents the false discovery rate (FDR) of 0.05. a PheWAS comparing African American SLE cases to African American controls. African Americans with SLE had more codes compared to matched controls for comorbidities across all organ systems, particularly cardiac and renal. The most significant codes for each organ system are labeled. b PheWAS comparing Caucasian SLE cases to Caucasian controls. Caucasians with SLE had more renal and SLE-related codes compared to controls. The most significant codes for organ systems are labeled 
age (53 \pm 16 vs. $53 \pm 16, p=0.86)$ and were predominantly female ( $90 \%$ vs. $90 \%, p=0.95)$. Using PheWAS to compare Caucasians with SLE to matched Caucasian controls, there were 262 codes that met the FDR of $5 \%$. Compared to the controls, the Caucasians with SLE had more codes that represented SLE-related and renal comorbidities (Fig. 2b, Additional file 1: Table S5). The most significant code was myalgia and myositis unspecified (OR $=8.30,95 \%$ CI $6.57-10.49$, FDR $p=2.90 \times 10^{-67}$ ), which signifies fibromyalgia in our EHR, based on a prior study [15]. Other significant codes included a dermatologic group of codes called erythematous conditions $(\mathrm{OR}=$ 14.01, 9.91-19.81, FDR $p=5.01 \times 10^{-48}$ ), which includes a code for discoid lupus, and renal failure $(\mathrm{OR}=6.16$, 4.80-7.91, FDR $p=7.91 \times 10^{-44}$ ). Other renal codes included nephritis; nephrosis; renal sclerosis (FDR $p=$ $4.88 \times 10^{-28}$ ) and chronic kidney disease (FDR $p=$ $3.17 \times 10^{-21}$ ). Notably, there were fewer cardiac and infection codes compared to the PheWAS of African Americans with SLE and African American controls. The most significant cardiac code was peripheral vascular disease $\left(\mathrm{OR}=8.19,5.40-12.42\right.$, FDR $\left.p=1.55 \times 10^{-21}\right)$. The most significant infection code was pneumonia $\left(\mathrm{OR}=3.26,2.37-12.42\right.$, FDR $\left.p=6.32 \times 10^{-12}\right)$. As expected, similar to the PheWAS of the African Americans with SLE and African American controls, codes that represent ACR SLE criteria [24] were more common in the Caucasians with SLE compared to the Caucasian controls. Significant codes included pain in joint, nephritis/nephrosis/renal sclerosis, thrombocytopenia, and pericarditis (Additional file 1: Table S5). In a sensitivity analysis, results remain unchanged when adjusted for years of EHR follow up.

\section{Conditional logistic regression models}

Using a conditional logistic regression model of SLE cases and matched controls (including both Caucasians and African Americans), we examined the association of SLE with PheWAS codes adjusting for age, race, and sex. SLE was significantly associated with hypertension $(\mathrm{OR}=2.18,95 \% 1.86-2.55, p<0.01)$, CHF $\left(\mathrm{OR}=2.33,1.73-3.13, p=2.2 \times 10^{-8}\right), \mathrm{CVD}(\mathrm{OR}=$ $\left.1.76,1.34-2.32, p=5.8 \times 10^{-5}\right)$, and cardiac dysrhythmias $\left(\mathrm{OR}=1.63,1.34-1.97, p=9.0 \times 10^{-7}\right)$ (Fig. 3, Additional file 1: Table S6). SLE was also independently associated with the following renal codes: chronic kidney disease $(\mathrm{OR}=7.04,5.73-8.65, p<$ $0.001)$, end-stage renal disease $(\mathrm{OR}=8.00,5.43-11.80$, $p<0.001)$, and renal transplant $(\mathrm{OR}=7.61,7.26-7.99$, $\left.p=1.1 \times 10^{-16}\right)$. Lastly, SLE disease status was independently associated with infections including pneumonia $(\mathrm{OR}=3.87,2.98-5.01, p<0.001)$ and sepsis/ bacteremia $(\mathrm{OR}=6.10,4.05-9.19, p<0.001)$.

\section{Discussion}

Using PheWAS in a large cohort of 1097 subjects with SLE using EHR data with decades of follow up, we uncovered an increased burden of comorbidities across all organ systems among African Americans compared to Caucasians with SLE. African Americans with SLE were two to four times more likely to have renal disease, cardiovascular disease, and infections. To the best of our knowledge, this is the first study to use PheWAS to examine racial disparities between African Americans and Caucasians with SLE. Since some comorbidities are more frequent in non-SLE African Americans compared with Caucasians [25], we determined the impact of SLE on comorbidities in African Americans. Compared to matched African American controls, African American subjects with SLE were significantly more likely to have comorbidities in all organ systems, notably in renal, cardiovascular, and infectious diseases.

PheWAS enables a systematic assessment of diverse phenotypes in the EHR, building upon both traditional cohort and administrative database studies. PheWAS has the potential to capture both SLE disease-related data such as ACR SLE criteria [24], as well as other comorbidities. Data on these comorbidities may not be collected in traditional cohort studies, while administrative database studies may not adequately capture SLE-related data. Further, administrative databases can have a fairly short duration of follow up [26, 27]. In contrast, our EHR has follow up over several decades with subjects with SLE having on average 9 years of follow up [20]. PheWAS has the power to capture diverse comorbidities in the EHR and uncover how these comorbidities contribute to racial disparities in SLE.

Compared to Caucasians, African American patients with SLE have increased renal disease. These disparities have been attributed to both genetic and non-genetic factors such as the environment and socioeconomic status [28]. As expected, we observed an increased burden of renal disease in African Americans compared to Caucasians with SLE, which agrees with findings in prior SLE cohorts [29-34]. While PheWAS confirmed known renal disparities in African Americans with SLE, it also uncovered an increased cardiovascular disease burden, which has not been previously well-described. African Americans with SLE were three times more likely to have CAD compared to Caucasians with SLE. Administrative studies have shown increased CAD in African Americans compared to Caucasians with SLE when restricting analyses to inpatient encounters and subsets of patients with SLE $[35,36]$. Our study builds upon these studies by including all patients with SLE and capturing CAD in both inpatient and outpatient encounters. In contrast to these administrative database studies, two cohort studies did not find increased CAD in African 


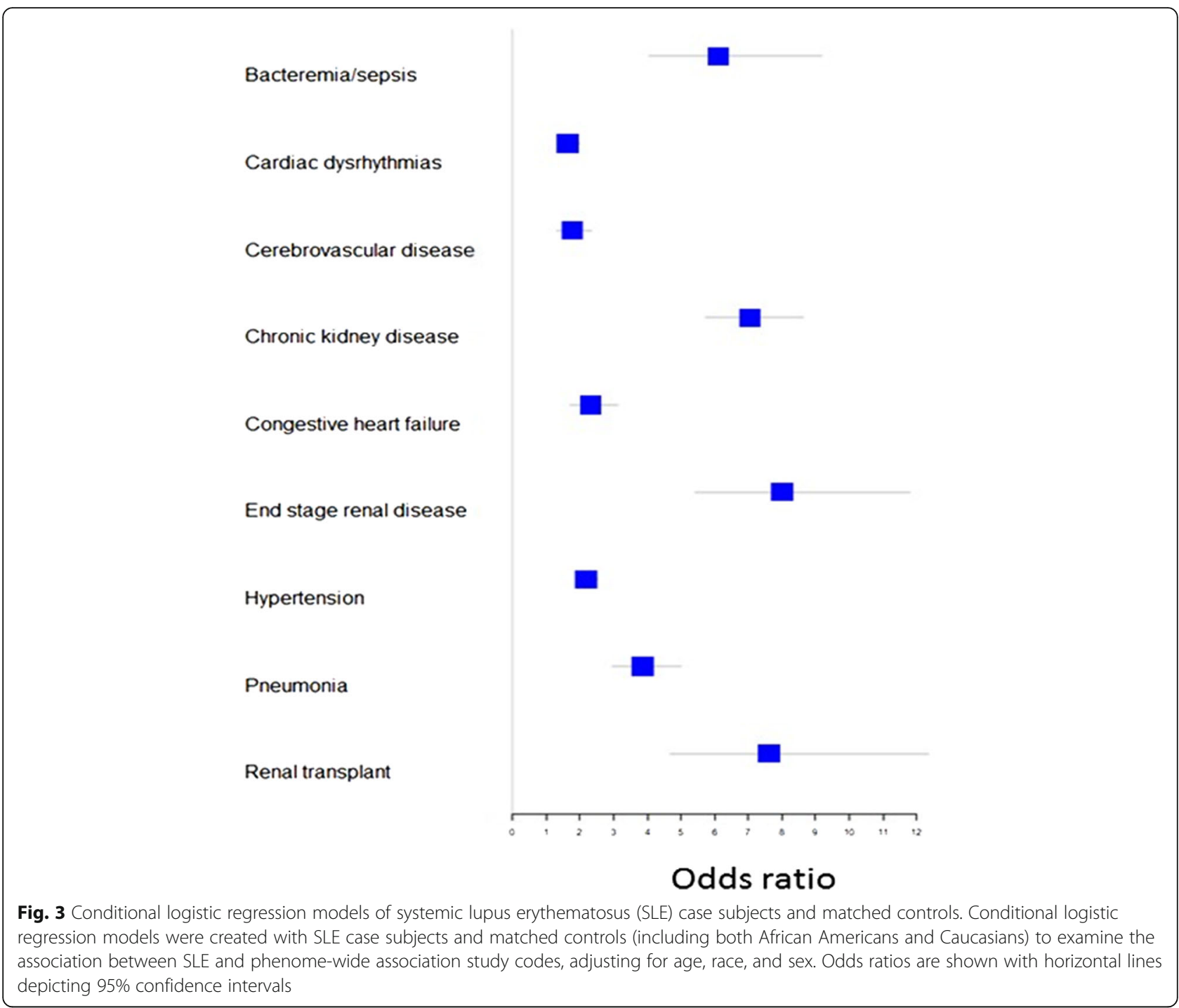

Americans compared to Caucasians with SLE [37-39]. These differences could be due to different SLE patient populations. In contrast to traditional SLE cohorts, our EHR SLE cohort may represent a more communitybased group of patients with SLE. Further, unless a cohort study collects data on a specific outcome, these outcomes may be underreported, as they may rely on either patient report or traditional methods that often focus on disease activity measures. These SLE cohorts also had a low frequency of CAD, myocardial infarction, and CVD events, with one study having only 34 patients with any vascular event [39]. These low-frequency events may have made these studies underpowered to detect differences in CAD in African Americans compared to Caucasians with SLE. In our EHR cohort, looking across multiple codes that captured CAD, we had 177 events.

In addition to CAD, African Americans with SLE were three times more likely to have CHF and CVD and more than four times more likely to have hypertension compared to Caucasians. There are fewer studies comparing risk of these cardiovascular diseases in African Americans to Caucasians with SLE, with mixed results $[36,37]$. Specifically, in one cohort, there were no differences in rates of CVD and PVD comparing African Americans to Caucasians [39]. This study included only 18 subjects with CVD and 5 with PVD in contrast to approximately 223 subjects with CVD and 25 with PVD in our study [39].

Beyond the increased renal and cardiac disease burden, African Americans with SLE had an increase in infectious diseases compared to Caucasians with SLE. African Americans were more than 3.5 times more likely to have pneumonia and twice as likely to have bacteremia and sepsis. Our study agrees with two studies using the Medicaid administrative database that identified an increased risk of serious infections in African 
Americans compared to Caucasians with SLE, with the most common being bacteremia, pneumonia, and cellulitis [26, 27]. Our study builds upon these studies by including both inpatient and outpatient infections and offering a longer follow up of 9 years compared to the mean follow up of the studies of 2.5 years.

To account for racial differences in comorbidities, we compared African Americans with SLE to matched African American controls, particularly since many of these comorbidities are more common in African Americans. As expected, African Americans with SLE had more codes related to ACR SLE criteria [24] showing that PheWAS can identify SLE disease characteristics in the EHR. Compared to matched controls, African Americans with SLE also had more comorbidities across all organ systems. Notably, African Americans with SLE were more likely to have codes for chronic kidney disease (CKD), end-stage renal disease (ESRD), and renal transplant. Using a conditional logistic regression model with SLE cases and matched controls (including both Caucasians and African Americans), adjusting for age, sex, and race, SLE remained independently associated with CKD, ESRD, and renal transplant suggesting that African American race was not the sole driver for increased renal disease.

Compared to matched controls, African Americans with SLE also had more codes for CAD, CVD, PVD, and arrhythmias. While a twofold to threefold increase in CAD has been described in subjects with SLE compared to population controls [40], there are few data comparing CAD events in African Americans with SLE compared to matched controls. One of the largest US population-based studies, the Nurses' Health study, compared rates of CAD in participants with and without SLE showing a twofold to threefold increase in CAD events [41]. Notably, the cohort was all female and 95\% Caucasian [41]. Our study is unique in that it included both male and female SLE patients and focused on African Americans. For other cardiac comorbidities, there are few studies comparing African American patients with SLE to matched controls [41, 42]. Further, studies often restrict analyses to subsets of patients with SLE $[41,42]$ while no studies directly compare patients with SLE to controls for PVD [43, 44] and AF. Our study builds upon large population-based studies by including male subjects and African Americans with SLE, who are often understudied and have adverse outcomes [45]. Our study also demonstrates novel findings of increased PVD and AF in African American patients with SLE compared to controls.

African Americans with SLE also had increased risk of multiple infections compared to matched controls. This increased risk of infection in SLE is likely due to immunosuppressant medications, the disease itself, or an interaction between these factors [46-48]. Two recent studies using the US Medicaid database investigated infection rates among different SLE patients but did not compare patients with SLE to matched controls [26, 27]. Our study establishes an increased risk of infection in African Americans with SLE compared to matched controls.

Our EHR-based PheWAS study has limitations. We used a previously validated algorithm to identify patients with SLE with a positive predictive value (PPV) of $89 \%$ and sensitivity of $86 \%$ [20]. Despite this algorithm's strong test characteristics, we may have captured some subjects who do not have a SLE diagnosis. Our clinical EHR data, in contrast to prospective cohort studies, does not contain disease activity and damage measures such as the Systemic Lupus Erythematosus Disease Activity Index (SLEDAI) [49] and Systemic Lupus International Collaborating Clinics Damage Index (SDI) [50], as these measures are not collected routinely in clinical practice. Thus, we cannot adjust for disease activity or damage in PheWAS. EHR-based algorithms that assess treatment response in inflammatory bowel disease [51] and CAD risk in inflammatory bowel disease and RA [52] have been created. Currently, however, there are no published, EHR-based algorithms assessing disease severity and activity in autoimmune diseases. Future directions include developing these algorithms in SLE. Next, this PheWAS was performed using billing codes at Vanderbilt only. Patients can receive care in multiple healthcare systems, which may not be documented in Vanderbilt's EHR. These potential missed diagnoses, however, would bias us to the null result. Missing data could be nonrandomly distribute, with more occurring in the controls in whom EHR follow up was shorter compared to patients with SLE. We adjusted for EHR follow-up time, which did not alter our main findings. Last, our study was performed using a single institution's EHR, potentially limiting generalizability of our results to other patients with SLE. Using an EHR-based cohort to study SLE, however, may capture a wider net of patients with SLE that are more representative of the community compared to patients with SLE recruited into a cohort. We did not have sufficient numbers of Hispanics or Asians, reflecting the demographics of middle Tennessee, to study patients with SLE with these ethnicities in our PheWAS. However, our EHR cohort included male subjects and African Americans with SLE, who are often understudied [45]. We acknowledge that the African American population in the USA is admixed, and these findings associated with the race construct could represent cultural and socioeconomic factors as well as genetic ancestry. Unfortunately, our de-identified resource does not contain socioeconomic data such as income level or insurance coverage, so we are unable to adjust for these factors in our PheWAS. 


\section{Conclusion}

Using PheWAS, we demonstrated an increased burden of comorbidities in African Americans with SLE compared to Caucasians with SLE and matched controls, including a spectrum of renal, cardiovascular, and infectious diseases. These findings suggest that clinicians managing patients with SLE should not only screen for SLE disease manifestations but also have suspicion of multiple cardiovascular and infectious diseases in their workup of common signs and symptoms to ensure appropriate and timely referrals and management. This high comorbidity burden in SLE, particularly in African Americans, argues for the need for access to care to not only rheumatology but also to primary and other subspecialty care. Further, this study demonstrates that an EHR-based approach can build upon traditional cohort and administrative database studies to examine racial disparities in SLE.

\section{Additional file}

Additional file 1: Table S1. Significant codes from the PheWAS of African Americans vs. Caucasians with SLE. Figure S1. Selected SLE disease criteria codes in the PheWAS of African Americans and Cauasians with SLE. Table S2. Selected SLE criteria codes from the PheWAS of African Americans and Caucasians with SLE. Table S3. Selected codes related to renal, cardiovascular disease, and infection from the PheWAS of African American SLE cases compared to matched African American controls. Table S4. Selected codes related to SLE criteria from the PheWAS of African American SLE cases and matched African American controls. Table S5. Selected codes from the PheWAS of Caucasian SLE cases compared to matched Caucasian controls. Table S6. Conditional logistic regression models with SLE cases and matched controls. (DOCX $134 \mathrm{~kb})$

\section{Abbreviations}

95\% Cl: 95\% Confidence intervals; ACR: American College of Rheumatology; AF: Atrial fibrillation; ANA: Anti-nuclear antibody; CAD: Coronary artery disease; CHF: Congestive heart failure; CKD: Chronic kidney disease; CVD: Cerebrovascular disease; EHR: Electronic health record; ESRD: End-stage renal disease; FDR: False discovery rate; GWAS: Genome-wide association study; OR: Odds ratio; PheWAS: Phenome-wide association study; PPV: Positive predictive value; PVD: Peripheral vascular disease; SDI: Systemic Lupus International Collaborating Clinics Damage Index; SLE: Systemic lupus erythematosus; SLEDAl: Systemic Lupus Erythematosus Disease Activity Index; VUMC: Vanderbilt University Medical Center

\section{Acknowledgements}

None.

\section{Funding}

This study was funded by the following grants: NIH/NICHD 5K12HD04348312 (Barnado), NCRR/NIH UL1 RR024975, NCATS/NIH ULTR000445, NLM R01LM010685 (Denny).

\section{Availability of data and materials}

The data used and analyzed during the current study are available from the corresponding author on reasonable request.

\section{Authors' contributions}

AB: literature search, study design, data collection, data analysis, data interpretation, manuscript preparation. RJC: study design, data collection, data analysis, data interpretation, manuscript preparation. CC: study design, data collection, manuscript preparation. LW: data analysis, data interpretation, manuscript preparation. JCD: study design, data analysis, data interpretation, manuscript preparation. LJC: study design, data analysis, data interpretation, manuscript preparation. All authors read and approved the final manuscript.

Ethics approval and consent to participate

Approval was obtained from the Institutional Review Board of Vanderbilt University Medical Center. As de-identified data were used, research was classified as non-human subject research, and informed consent was waived.

\section{Consent for publication}

Not applicable.

\section{Competing interests}

The authors declare that they have no competing interests.

\section{Publisher's Note}

Springer Nature remains neutral with regard to jurisdictional claims in published maps and institutional affiliations.

\section{Author details}

${ }^{1}$ Department of Medicine, Vanderbilt University Medical Center, 1161 21st Avenue South, T3113 MCN, Nashville, TN 37232, USA. ${ }^{2}$ Department of Biomedical Informatics, Vanderbilt University Medical Center, Nashville, TN, USA. ${ }^{3}$ Department of Medicine, Lehigh Valley Health Network, Allentown, PA, USA. ${ }^{4}$ Department of Dermatology, Vanderbilt University Medical Center, Nashville, TN, USA.

Received: 23 January 2018 Accepted: 6 March 2018

Published online: 10 April 2018

\section{References}

1. Sundquist J, Johansson SE. The influence of socioeconomic status, ethnicity and lifestyle on body mass index in a longitudinal study. Int J Epidemiol. 1998:27:57-63.

2. Demas KL, Costenbader KH. Disparities in lupus care and outcomes. Curr Opin Rheumatol. 2009;21:102-9.

3. Lim SS, Bayakly AR, Helmick CG, Gordon C, Easley KA, Drenkard C. The incidence and prevalence of systemic lupus erythematosus, 2002-2004: the Georgia Lupus Registry. Arthritis Rheum. 2014;66:357-68.

4. Somers EC, Marder W, Cagnoli P, Lewis EE, DeGuire P, Gordon C, et al. Population-based incidence and prevalence of systemic lupus erythematosus: the Michigan Lupus Epidemiology and Surveillance program. Arthritis Rheum. 2014;66:369-78.

5. Centers for Disease C, Prevention. Trends in deaths from systemic lupus erythematosus-United States, 1979-1998. MMWR Morb Mortal Wkly Rep. 2002;51:371-4.

6. Krishnan E, Hubert HB. Ethnicity and mortality from systemic lupus erythematosus in the US. Ann Rheum Dis. 2006;65:1500-5.

7. Pathak J, Kho AN, Denny JC. Electronic health records-driven phenotyping: challenges, recent advances, and perspectives. J Am Med Inform Assoc. 2013;20:e206-11.

8. Ritchie MD, Denny JC, Crawford DC, Ramirez AH, Weiner JB, Pulley JM, et al. Robust replication of genotype-phenotype associations across multiple diseases in an electronic medical record. Am J Hum Genet. 2010;86:560-72.

9. Bowton E, Field JR, Wang S, Schildcrout JS, Van Driest SL, Delaney JT, et al. Biobanks and electronic medical records: enabling cost-effective research. Sci Transl Med. 2014;6:234cm3.

10. Denny JC, Ritchie MD, Basford MA, Pulley JM, Bastarache L, Brown-Gentry K, et al. PheWAS: demonstrating the feasibility of a phenome-wide scan to discover gene-disease associations. Bioinformatics. 2010;26:1205-10.

11. Denny JC, Bastarache L, Ritchie MD, Carroll RJ, Zink R, Mosley JD, et al. Systematic comparison of phenome-wide association study of electronic medical record data and genome-wide association study data. Nat Biotechnol. 2013:31:1102-10.

12. Denny JC, Crawford DC, Ritchie MD, Bielinski SJ, Basford MA, Bradford Y, et al. Variants near FOXE1 are associated with hypothyroidism and other thyroid conditions: using electronic medical records for genome- and phenome-wide studies. Am J Hum Genet. 2011;89:529-42.

13. Hebbring SJ, Schrodi SJ, Ye Z, Zhou Z, Page D, Brilliant MH. A PheWAS approach in studying HLA-DRB1*1501. Genes Immun. 2013;14:187-91.

14. Liao KP, Kurreeman F, Li G, Duclos G, Murphy S, Guzman R, et al. Associations of autoantibodies, autoimmune risk alleles, and clinical 
diagnoses from the electronic medical records in rheumatoid arthritis cases and non-rheumatoid arthritis controls. Arthritis Rheum. 2013:65:571-81.

15. Doss J, Mo H, Carroll RJ, Crofford LJ, Denny JC. Phenome-wide association study of rheumatoid arthritis subgroups identifies association between seronegative disease and fibromyalgia. Arthritis Rheum. 2017;69:291-300.

16. Liao KP, Sparks JA, Hejblum BP, Kuo IH, Cui J, Lahey L, et al. Phenome-wide association study of autoantibodies to citrullinated and non-citrullinated epitopes in rheumatoid arthritis. Arthritis Rheum. 2017;69:742-9.

17. Denny JC, Bastarache L, Roden DM. Phenome-wide association studies as a tool to advance precision medicine. Annu Rev Genomics Hum Genet. 2016;17:353-73.

18. Bush WS, Oetjens MT, Crawford DC. Unravelling the human genomephenome relationship using phenome-wide association studies. Nat Rev Genet. 2016;17:129-45.

19. Roden DM, Pulley JM, Basford MA, Bernard GR, Clayton EW, Balser JR, et al. Development of a large-scale de-identified DNA biobank to enable personalized medicine. Clin Pharmacol Ther. 2008;84:362-9.

20. Barnado A, Casey C, Carroll RJ, Wheless L, Denny JC, Crofford LJ. Developing electronic health record algorithms that accurately identify patients with systemic lupus erythematosus. Arthritis Care Res. 2017;69:687-93.

21. Schildcrout JS, Denny JC, Bowton E, Gregg W, Pulley JM, Basford MA, et al. Optimizing drug outcomes through pharmacogenetics: a case for preemptive genotyping. Clin Pharmacol Ther. 2012;92:235-42.

22. Dumitrescu L, Ritchie MD, Brown-Gentry K, Pulley JM, Basford M, Denny JC, et al. Assessing the accuracy of observer-reported ancestry in a biorepository linked to electronic medical records. Genet Med. 2010;12:648-50.

23. Carroll RJ, Bastarache L, Denny JC. R PheWAS: data analysis and plotting tools for phenome-wide association studies in the R environment. Bioinformatics. 2014;30:2375-6.

24. Hochberg MC. Updating the American College of Rheumatology revised criteria for the classification of systemic lupus erythematosus. Arthritis Rheum. 1997:40:1725.

25. Mozaffarian D, Benjamin EJ, Go AS, Arnett DK, Blaha MJ, Cushman M, et al. Heart disease and stroke statistics-2016 update: a report from the American Heart Association. Circulation. 2016;133:e38-360.

26. Feldman CH, Hiraki LT, Winkelmayer WC, Marty FM, Franklin JM, Kim SC, et al. Serious infections among adult Medicaid beneficiaries with systemic lupus erythematosus and lupus nephritis. Arthritis Rheum. 2015;67:1577-85.

27. Hiraki LT, Feldman CH, Marty FM, Winkelmayer WC, Guan H, Costenbader $\mathrm{KH}$. Serious infection rates among children with systemic lupus erythematosus enrolled in Medicaid. Arthritis Care Res. 2017;69:1620-6.

28. Gonzalez LA, Toloza SM, McGwin G Jr, Alarcon GS. Ethnicity in systemic lupus erythematosus (SLE): its influence on susceptibility and outcomes. Lupus. 2013;22:1214-24.

29. Fernandez M, Alarcon GS, Calvo-Alen J, Andrade R, McGwin G Jr, Vila LM, et al. A multiethnic, multicenter cohort of patients with systemic lupus erythematosus (SLE) as a model for the study of ethnic disparities in SLE. Arthritis Rheum. 2007;57:576-84.

30. Alarcon GS, McGwin G Jr, Petri M, Ramsey-Goldman R, Fessler BJ, Vila LM, et al. Time to renal disease and end-stage renal disease in PROFILE: a multiethnic lupus cohort. PLoS Med. 2006;e396:3.

31. Burgos PI, McGwin G Jr, Pons-Estel GJ, Reveille JD, Alarcon GS, Vila LM. US patients of Hispanic and African ancestry develop lupus nephritis early in the disease course: data from LUMINA, a multiethnic US cohort (LUMINA LXXIV). Ann Rheum Dis. 2011;70:393-4.

32. Korbet SM, Schwartz MM, Evans J, Lewis EJ, Collaborative Study G. Severe lupus nephritis: racial differences in presentation and outcome. J Am Soc Nephrol. 2007;18:244-54

33. Hiraki LT, Lu B, Alexander SR, Shaykevich T, Alarcon GS, Solomon DH, et al. End-stage renal disease due to lupus nephritis among children in the US, 1995-2006. Arthritis Rheum. 2011;63:1988-97.

34. Shaharir SS, Mustafar R, Mohd R, Mohd Said MS, Gafor HA. Persistent hypertension in lupus nephritis and the associated risk factors. Clin Rheumatol. 2015:34:93-7.

35. Scalzi LV, Hollenbeak CS, Wang L. Racial disparities in age at time of cardiovascular events and cardiovascular-related death in patients with systemic lupus erythematosus. Arthritis Rheum. 2010;62:2767-75.

36. Gomez-Puerta JA, Feldman CH, Alarcon GS, Guan H, Winkelmayer WC, Costenbader $\mathrm{KH}$. Racial and ethnic differences in mortality and cardiovascular events among patients with end-stage renal disease due to lupus nephritis. Arthritis Care Res. 2015:67:1453-62.
37. Maynard JW, Fang H, Petri M. Low socioeconomic status is associated with cardiovascular risk factors and outcomes in systemic lupus erythematosus. J Rheumatol. 2012;39:777-83.

38. Petri M, Perez-Gutthann S, Spence D, Hochberg MC. Risk factors for coronary artery disease in patients with systemic lupus erythematosus. Am J Med. 1992;93:513-9.

39. Toloza SM, Uribe AG, McGwin G Jr, Alarcon GS, Fessler BJ, Bastian HM, et al. Systemic lupus erythematosus in a multiethnic US cohort (LUMINA). XXIII. Baseline predictors of vascular events. Arthritis Rheum. 2004;50:3947-57.

40. Schoenfeld SR, Kasturi S, Costenbader KH. The epidemiology of atherosclerotic cardiovascular disease among patients with SLE: a systematic review. Semin Arthritis Rheum. 2013;43:77-95.

41. Hak AE, Karlson EW, Feskanich D, Stampfer MJ, Costenbader KH. Systemic lupus erythematosus and the risk of cardiovascular disease: results from the nurses' health study. Arthritis Rheum. 2009:61:1396-402.

42. Ward MM. Premature morbidity from cardiovascular and cerebrovascular diseases in women with systemic lupus erythematosus. Arthritis Rheum. 1999;42:338-46.

43. Urowitz MB, Gladman D, Ibanez D, Bae SC, Sanchez-Guerrero J, Gordon C, et al. Atherosclerotic vascular events in a multinational inception cohort of systemic lupus erythematosus. Arthritis Care Res. 2010;62:881-7.

44. Burgos PI, Vila LM, Reveille JD, Alarcon GS. Peripheral vascular damage in systemic lupus erythematosus: data from LUMINA, a large multi-ethnic U.S. cohort (LXIX). Lupus. 2009:18:1303-8.

45. Tan TC, Fang H, Magder LS, Petri MA. Differences between male and female systemic lupus erythematosus in a multiethnic population. J Rheumatol. 2012;39:759-69.

46. Gladman DD, Hussain F, Ibanez D, Urowitz MB. The nature and outcome of infection in systemic lupus erythematosus. Lupus. 2002;11:234-9.

47. Ginzler E, Diamond H, Kaplan D, Weiner M, Schlesinger M, Seleznick M. Computer analysis of factors influencing frequency of infection in systemic lupus erythematosus. Arthritis Rheum. 1978;21:37-44.

48. Bosch X, Guilabert A, Pallares L, Cerveral R, Ramos-Casals M, Bove A, et al. Infections in systemic lupus erythematosus: a prospective and controlled study of 110 patients. Lupus. 2006:15:584-9.

49. Bombardier C, Gladman DD, Urowitz MB, Caron D, Chang CH. Derivation of the SLEDAI. A disease activity index for lupus patients. The Committee on Prognosis Studies in SLE. Arthritis Rheum. 1992;35:630-40.

50. Gladman D, Ginzler E, Goldsmith C, Fortin P, Liang M, Urowitz M, et al. The development and initial validation of the Systemic Lupus Internationa Collaborating Clinics/American College of Rheumatology damage index for systemic lupus erythematosus. Arthritis Rheum. 1996;39:363-9.

51. Ananthakrishnan AN, Cagan A, Cai T, Gainer VS, Shaw SY, Savova G, et al. Identification of nonresponse to treatment using narrative data in an electronic health record inflammatory bowel disease cohort. Inflamm Bowel Dis. 2016:22:151-8.

52. Liao KP, Ananthakrishnan AN, Kumar V, Xia Z, Cagan A, Gainer VS, et al. Methods to develop an electronic medical record phenotype algorithm to copare the risk of coronary artery disease across 3 chronic disease cohorts. PLoS One $2015 \cdot 10: 8$

\section{Submit your next manuscript to BioMed Central and we will help you at every step:}

- We accept pre-submission inquiries

- Our selector tool helps you to find the most relevant journal

- We provide round the clock customer support

- Convenient online submission

- Thorough peer review

- Inclusion in PubMed and all major indexing services

- Maximum visibility for your research

Submit your manuscript at www.biomedcentral.com/submit 